\title{
Cooperativas agropecuarias. Un factor indispensable para la soberanía alimentaria en Cuba
}

\author{
(Agricultural Cooperatives. \\ An Indispensable Factor for Food Sovereignty in Cuba)
}

Yaumara Acosta Morales ${ }^{1}$

Universidad de Cienfuegos, Cuba

Sumario: I. Introducción. II. Cooperativismo internacional. III. El movimiento Cooperativo en Cuba. IV. La Unidades Básicas de Producción Cooperativa. V. El cooperativismo en la coyuntura actual. VI. Conclusiones.

Resumen. Las cooperativas agropecuarias son la herramienta comúnmente utilizada a nivel mundial para promover procesos de integración. La globalización de los mercados y el aumento maximizado de la competencia hacen precisa una vertiginosa y eficiente respuesta en el cooperativismo internacional. Las decisiones arrogadas por las sociedades cooperativas han ido orientadas a la ejecución de procesos de integración o concentración, siendo la constitución de cooperativas de segundo grado una de las vías más estiladas. Las Cooperativas agropecuarias son empresas vinculadas de forma estable al territorio rural y al productor agroalimentario. Además, ejercen el liderazgo en la economía de estas áreas y en la fijación de población en municipios rurales, contribuyendo así al equilibrio, la ordenación y el desarrollo rural.

Palabras clave: cooperativas agropecuarias; soberanía alimentaria; cooperativismo internacional.

Abstract. Agricultural cooperatives are the tool commonly used worldwide to promote integration processes. The globalization of markets and the maximized increase in competition require a dizzying and efficient response in international cooperativism. The decisions made by cooperative societies have been oriented towards the execution of integration or concentration processes, with the constitution of second-degree cooperatives being one of the most popular routes. Agricultural cooperatives are companies linked in a stable

1 Master en Dirección. Profesora Auxiliar del Departamento de Ciencias Sociales de la Universidad de Cienfuegos, Cuba. E-mail: yamorales@ucf.edu.cu 
way to the rural territory and the agri-food producer. In addition, they exercise leadership in the economy of these areas and in the establishment of population in rural municipalities, thus contributing to balance, management and rural development.

Keywords: Agricultural cooperatives; food sovereignty; international cooperativism. 


\section{Introducción}

La cooperación es un proceso inacabado, continuo, incesante. La cooperación puede abarcar procesos temporales o procesos permanentes sin dar lugar a una forma organizativa económico y social concreta y jurídicamente independientes o dando lugar a una organización económico-social jurídicamente independiente y autónoma.

La cooperación en el proceso de trabajo, que es la forma imperante en los comienzos de la civilización, en los pueblos de cazadores, o en la agricultura de las comunidades indias se basa, de una parte, en la propiedad colectiva sobre las condiciones de producción y de otra parte en el hecho de que el individuo no ha roto todavía el cordón umbilical que le une a la comunidad o a la tribu, de la que forma parte como la abeja de la colmena. ${ }^{2}$

En esta fase precursora del cooperativismo es necesario destacar de manera especial al destacado ideólogo Robert Owen (1771-1858), quien identifica el término cooperación como sinónimo de socialismo, distanciándolo del sistema individualista de la libre competencia ${ }^{3}$. Owen el autodidacta, industrial afortunado desde muy joven, innovador en técnicas y sistemas sociales, en el furor de la revolución industrial, intentó llevar a la práctica sus ideas organizando las colonias de Armony Hall en su propio país Inglaterra y la de Nueva Armonía en Estados Unidos (Indiana); la bolsa de trabajo y las instituciones sindicales de alcance nacional. ${ }^{4}$

La constitución de la Rochdale Society of Equitable Pioneers en Inglaterra en 1844, fue el comienzo del movimiento cooperativo sistematizado, su progreso fue lento, pero demostró en momentos de penuria que la cooperación es enemiga de la pobreza. Los principios fundamentales fueron: independencia económica, devolución de los excedente ahorrados (retorno cooperativo), democracia: un voto por persona incluyendo las mujeres, educación cooperativa (para la que destinaron el 2,5\% de los ahorros). Desde 1844 a 1934 habían distribuido excedentes ahorrados por un valor de 400 mil libras $^{5}$

2 Marx, C.: Obras escogidas, T II. Ed. Progreso. Moscú. 1989.

3 Fernández Peiso, A.: La Cooperativa base para, su legislación en Cuba. Ciencias Sociales. Cuba. 2012. p. 7.

4 Miranda Lorenzo H.: Cooperativismo y autogestión en la visión de Marx, Engels y Lenin. Parte 2. TEMA 3. Las cooperativas y los pensadores socialistas. Cooperativas y socialismo. Una mirada desde Cuba. 2012.

5 Fernández Peiso: A op. cit., 13. 
Una cooperativa es una sociedad económica creada con la unión de los recursos de sus miembros y conducida conjuntamente con estos, de modo democrático. Las organizaciones cooperativas se pueden fundar con la unión de gran diversidad de recursos - monetarios, materiales, de equipo, de materia prima, de mano de obra-y en las más disimiles esferas de actividades; se hacen para satisfacer las necesidades y resolver los diferentes problemas de sus miembros.

Su objeto es social no lucrativo, y se guía por los principios que a continuación se anuncia: membresía abierta y voluntaria, control democrático de los miembros, participación económica de los socios, autonomía e independencia, educación, entrenamiento e información, cooperación entre cooperativas, compromiso con la comunidad.

\section{Cooperativismo internacional}

El 7 de febrero de 1995 en la Asamblea General de la Naciones Unidas en su cuadragésimo noveno período de sesiones el tema 95 del programa aprobó la resolución 49/155. El papel de las cooperativas habida cuenta de las nuevas tendencias económicas y sociales, donde se reconoce que las cooperativas, en sus distintas formas, están pasando a ser un factor indispensable del desarrollo económico y social de todos los países y promueven la participación más plena posible en el proceso de desarrollo de todos los grupos de la población, inclusive las mujeres, los jóvenes, las personas discapacitadas y los ancianos. ${ }^{6}$

Las cooperativas mundialmente se ocupan en una gran diversidad de actividades. En la zona occidental de Europa se comercializa a través de las cooperativas el $60 \%$ de los productos de orígenes agropecuarios; en Noruega y Suecia, las de consumo controlan el $25 \%$ y el $17 \%$ el mercado, respectivamente. Las cooperativas en Finlandia producen el $96 \%$ de los lácteos. El $50 \%$ de los huevos y el $34 \%$ de los productos forestales. El $22 \%$ del Producto Interno Bruto (PIB) de Nueva Zelanda era generado por estas organizaciones en la década pasada. ${ }^{7}$ Actualmente la Corporación Mondragón es el mayor grupo

6 Resolución aprobada por la Asamblea General de las Naciones Unidas [sobre la base del informe de la Tercera Comisión (A/49/605)] 49/155. El papel de las cooperativas habida cuenta de las nuevas tendencias económicas y sociales.

7 Cruz Reyes J. y Piñeiro Harnecker C.: La introducción a las cooperativas. Editorial Caminos, Cuba, 2012. p. 31. 
cooperativista del mundo, así como el primero del País Vasco y el séptimo de España, compuesta por más de 100 cooperativas de actividades diversas. ${ }^{8}$

En Canadá el 33\% de la población es miembro de una cooperativa. Las cooperativas desempeñan un papel fundamental en el sector agrícola de la economía canadiense, especialmente en la transformación y comercialización de cereales y oleaginosas, leche y productos lácteos, aves, miel, derivados del árbol de arce, frutas, legumbres y ganado. Específicamente en Quebec, las cooperativas ascienden a 3.380 unidades empresariales, integradas por 7.200 .000 socios (aproximadamente el $70 \%$ de la población), generando 75.000 empleos y movilizando activos que bordean los US $\$ 83.000$ millones. $^{9}$

Argentina posee más de 20.000 cooperativas de diferentes tipos: agropecuarias, de vivienda, de consumo, de crédito, de provisión, de servicios públicos, de trabajo, etc. Así también en Argentina, desde la segunda mitad de la década del 2000 se comenzaron a gestar muchas empresas recuperadas por sus trabajadores, quienes las ocuparon y pusieron a funcionar luego de su abandono por los accionistas de las mismas. Estas empresas (principalmente fábricas) en su mayoría han preferido convertirse en cooperativas autogestionadas. ${ }^{10}$

En Chile existen una gran cantidad de pequeñas y medianas empresas cooperativas en el mundo agrícola y ganadero. Suelen recibir apoyo estatal a través del Instituto Nacional de Desarrollo Agropecuario. Las cooperativas de vivienda chilenas también desarrollan una actividad importante en el ámbito de la vivienda adquirida con subsidio habitacional que entrega el Estado chileno.

En América Latina el cooperativismo comenzó a finales del siglo XIX y principios del siglo $x x$, gracias a los inmigrantes provenientes de Francia, Italia, Alemania e Inglaterra, quienes gracias a su experiencia y conocimiento en el tema de cooperativismo lograron llegar a países como Argentina, Brasil, Uruguay y Paraguay e incursionar allí con cooperativas de comercialización y suministros agrícolas, cooperativas de crédito, consumo y vivienda. Este sector se fortalece en el siglo xx debido a la

8 Altuna Gabilondo L.; Loyola Idiakez A.; Pagalday Tricio E.: Mondragón: Ios dilemas de un cooperativismo maduro. Editorial Caminos, Cuba, 2012, p. 191.

9 Confederación de Cooperativas de Colombia: Cooperativas en el mundo. Canadá. https://confecoop.coop/cooperativismo/en-el-mundo/canada/

10 Hudson, J. P.: «Políticas públicas y empresas recuperadas por sus obreros en Argentina: un análisis del Programa de Trabajo Autogestionado». 2004-2012. Apuntes [online]. Vol. 43, No. 79, pp. 157-184. 2016, 
crisis económica de 1930, debido a la acción de la iglesia, el gobierno, los movimientos sociales y políticos, entre otros ${ }^{11}$.

Gracias a los programas de ayuda como la «Alianza para el progreso», de la agencia internacional de Estados Unidos, orientados a la promoción y el financiamiento de cooperativas agrarias, de ahorro, crédito y vivienda; el movimiento cooperativo presenta entre 1960 y 1980 una duplicación en el número de cooperativas, activos, cobertura social y patrimonio, según datos de la Organización Cooperativa de las Américas. ${ }^{12}$

La propiedad cooperativa que se fomenta durante la construcción socialista, por su condición de propiedad colectiva, es considerada una forma de propiedad socialista. Hasta ahora a pesar de sus resultados productivos y sociales, en Cuba la forma cooperativa estuvo limitada a la producción agropecuaria ${ }^{13}$.

Las ideas acerca del cooperativismo forman parte de la ideología política de la Revolución Cubana y su portador principal ha sido el compañero Fidel. Baste recordar su alegato en El Moncada, pasando por sus llamados a la cooperación entre los campesinos de la Sierra Maestra, hasta su introducción en el texto de la Primera Ley de Reforma Agraria luego de una rápida lectura durante el vuelo de aviación a Santiago de Cuba camino de La Plata donde sería firmada por el gobierno en pleno.

\section{El movimiento cooperativo en Cuba}

El cooperativismo agrícola en Cuba es históricamente hablando un fruto autóctono de la Revolución. El capitalismo dependiente en la etapa neocolonial nunca le dio cabida a pesar de esfuerzos aislados por promoverlo. Las ideas acerca del cooperativismo forman parte de la ideología política de la Revolución Cubana y su portador principal ha sido el compañero Fidel. Baste recordar su alegato en El Moncada, pasando por sus llamados a la cooperación entre los campesinos de la Sierra Maestra, hasta su introducción en el texto de la Primera Ley de

11 Análisis del modelo cooperativo en el nuevo escenario económico (primer taller ideológico). Panamá, 10 y 11 de junio del 2004.

12 Pineda Suárez, C.J.; Pardo Martínez, E. y Castillo Soto, M.E.: Palacios Villegas, N. Cooperativismo mundial 150 años. Consult America Grupo Asesor. Bogotá, Colombia. 1994.

13 Fernández Peiso, A.: La Cooperativa. Bases para su legislación en Cuba. La Habana: Ciencias Sociales. 2012. 
Reforma Agraria luego de una rápida lectura durante el vuelo de aviación a Santiago de Cuba camino de La Plata donde sería firmada por el gobierno en pleno. Luego de varios experimentos cooperativistas en los años sesenta y primera mitad de los setenta, el cooperativismo se erigiría en el núcleo central de la política agrocampesina para el completo desarrollo del socialismo en el medio rural a partir de la segunda mitad de los años setenta. ${ }^{14}$

A medida que se desarrolla el país en el ámbito de la agricultura va ganando autonomía la mujer en el transcurso de los años de la revolución la mujer cubana ha logrado emanciparse, las mujeres en la actualidad pueden solicitar tierras para trabajarla, formando parte así del movimiento de mujeres líderes que integran este proyecto de la ANAP. También abarca el liderazgo femenino la agricultura en todas sus manifestaciones, en el desarrollo del ganado menor y hasta el mayor.

Aunque se ha ganado terreno aún falta promoción a estas actividades que antes eran exclusivamente de los hombres y para ello es necesaria la colaboración no solo de la FMC que siempre ha estado presente sino también de otras organizaciones de masa. No obstante se visualiza la autonomía de la mujer en el mercado. Por otra parte los directivos de la ANAP, la FMC y miembros del gobierno realizan visitas a los usufructuarios, de los cuales un porciento son féminas, manteniéndose una relación armónica entre la dirección y la base. Cumpliéndose así con el decreto 300, 304 y 19 vinculados a las UBPC y las CPA para atender a los usufructuarios y que sean respaldados jurídicamente.

En cuanto las posibilidades reales de financiamiento y relaciones con el banco, aquellas personas que solicitan las tierras pueden pedir un crédito de hasta 20.000 pesos para limpiar el área que desea trabajar, con facilidades de pago. Se controla que se controla que desde el preciso momento que se hace la solicitud y los sujetos pasan a ser usufructuarios se le exige el cumplimiento del contrato si el mismo puede extinguírsele y perder las tierras.

Existe una cultura de género en las organizaciones, las mujeres son respetadas por sus compañeros masculinos, son reconocidas y las ganancias percibidas por la labor que se realiza son sin distinción de género, tienen derecho al descanso a licencia en caso de enfermedad o de embarazo. La ANAP y la FMC tienen programas de superación tanto

14 Villareal Domínguez J.: Fundamentos teóricos y metodológicos sobre la reconversión productiva en el sector cooperativo cubano, consultado en https://www. monografias.com/trabajos69/reconversion-productiva-sector-cooperativo-canero/ reconversion-productiva-sector-cooperativo-canero2.shtml 
para obreros que quieran alcanzar el nivel superior como directivos que deseen una superación general y cultural. ${ }^{15}$

Se necesita promover más las nuevas oportunidades que existen para la entrega de tierras, la solicitud del crédito y sobre todo, hacerlo extensivo para aquellas mujeres emprendedoras, que logren el empoderamiento que exigen los tiempo y el lugar que ha conquistado con el sudor de su trabajo, entrega y sacrificio.

Se impone la necesidad de rescatar la integración necesaria entre la agroindustria de la caña de azúcar y la base de producción de alimento del ganado vacuno y porcino, así como la siembra de caña destinada directamente a la alimentación del ganado y base forrajera.

Indudablemente, en el país no existía un desarrollado sistema de organismos que hicieran posible el acceso a crédito agrícola ni una desarrollada red de venta de insumos para el sector agrícola. Esta insuficiencia, conllevó a la creación de la Asociación Nacional de Agricultores Pequeños (ANAP), en el año 1961, resultó un viraje cualitativo en las relaciones económicas y políticas estado-campesinos. Esta organización organizó y ayudó a unir a la mayoría de los agricultores pequeños en células primarias, las Asociaciones Campesinas, con una disposición semejante a las de una cooperativa. Dichas agrupaciones o asociaciones de base se convirtieron en entidades formadoras de colectivismo y en la clave del enlace para que el estado organizara su apoyo material, técnico y financiero a los productores privados y coordinara los acopios de estos productores. Transitoriamente, la ANAP asumió la organización de los suministros y créditos a los agricultores pequeños en el período 1961-1963, a petición del Gobierno revolucionario. Y es precisamente en este tiempo en que se forjan formas muy avanzadas de colaboración e integración entre los campesinos: brigadas de ayuda mutua, Cooperativas de Créditos y Servicios (Ilamadas CCS, con propiedad común sobre algunos medios de producción reproducibles) y las Sociedades Agropecuarias, con la colectivización de la tierra y otros recursos. ${ }^{16}$

El movimiento cooperativo abarcó a las grandes masas campesinas; más de un tercio se integró a las llamadas Cooperativas de Producción Agropecuaria (CPA) con alrededor del $50 \%$ del fondo de tierras del sector campesino. Hacia finales de los ochenta y hasta la fecha el sec-

15 Valdés Paz J.: Los procesos de organización agraria en Cuba 1959-2006. Fundación Antonio Nuñez Jiménez de la Naturaleza y el hombre, Cuba, 2010.

16 Cruz Reyes, J.: «Sobre cooperativas agrícolas de segundo grado: experiencias cubanas y consideraciones hacia el futuro». Economía y Desarrollo, vol. 158, núm. 1, enero-junio, Universidad de La Habana La Habana, Cuba, 2017, pp. 225-244. 
tor cooperativo CPA ocupa cerca del $10 \%$ del fondo de tierras del país, con casi 1200 entidades y más de 60 mil asociados ${ }^{17}$.

Entre 1976-1978 la Dirección de Atención al Sector Campesino (DASC) en el seno del MINAG y en sus subdirecciones administrativas en provincias y municipios con estructuras homólogas. Cuyas funciones era la de planificación de la producción y los abastecimientos, el control, asesoría y cumplimiento de la legislación agraria en el sector campesino y las relaciones contractuales con las empresas estatales, etcétera ${ }^{18}$. Un elemento clave de estos cambios fue la inclusión de la producción campesina en el sistema de planificación de la economía nacional a través del MINAG y con la activa participación de la ANAP a los niveles correspondientes. Este cambio significó predeterminar con tiempo suficiente el volumen de producción a contratar con cada productor y cooperativa, y nominalizar los recursos, aseguramientos y servicios técnico-productivos correspondientes a ellos. También fueron creadas Empresas Provinciales de Aseguramientos al Sector Campesino (EASC) que, a través de sus establecimientos municipales, se especializarían en la comercialización de los insumos y la realización de otros aseguramientos a los campesinos y CPA. ${ }^{19}$

Su participación en la producción nacional rebasa en mucho el volumen relativo de tierra agrícola en posesión. La economía colectiva demostró durante todos estos años su vitalidad y viabilidad como forma de producción y de vida, aún en los peores momentos del ciclo económico cubano a finales de los ochenta y primera mitad de los noventa, a pesar de los errores y tendencias negativas e insuficiencias que ha padecido; además evidenció su capacidad para la sobrevivencia y las posibilidades y potencialidades del trabajo cooperado y combinado como una forma socialista eficiente de economía y de vida en el medio rural. La confirmación más plena de esta tesis se validó en el primer tercio de los noventa cuando sirvió de referente para los cambios en la tenencia de la tierra y en la organización de las nuevas formas de producción a partir de las entidades estatales agropecuarias; concretamente el modelo UBPC emerge tomando la experiencia de las CPA.

El IV Congreso del Partido Comunista de Cuba, en las ya duras y críticas circunstancias de 1991, casi tres meses antes del colapso de la URSS, en su Resolución Económica y en otros acuerdos sentó las bases políticas y organizacionales para enfrentar dinámicamente los cambios

17 Nova, A.: Las cooperativas agropecuarias en Cuba: 1959-presente. Editorial Camino. 2012. p. 321.

18 Cruz Reyes, J.: op. cit, 231.

19 Ibidem. 
y ajustes necesarios según el curso de los acontecimientos internacionales e internos. La reforma en 1992 a la Constitución de 1976 abriría los espacios legales para la apertura, los ajustes y cambios estructurales que se introducirían en el primer tercio de los años 90 para enfrentar la crisis económica y agroalimentaria más profunda de la historia revolucionaria.

El V Congreso del Partido Comunista de Cuba, celebrado en 1997, marcó un importante hito en la historia del país al dar continuidad a la política de cambios enfilados a garantizar la construcción socialista en las nuevas circunstancias históricas.

La vertiente agraria de la reforma en particular ha generado cambios sustanciales en la estructura de la tenencia y explotación del suelo. La ampliación del cooperativismo con el surgimiento de las Unidades Básicas de Producción Cooperativa (UBPC) a partir de las granjas estatales forma parte de su contenido esencial. Otra de sus aristas principales es el perfeccionamiento constante del sector estatal y la consolidación de las Cooperativas de Producción Agropecuaria (CPA). Además, se ha extendido la producción individual con la entrega de tierras en usufructo; se han fortalecido a su vez las Cooperativas de Créditos y Servicios (CCS) y la apertura a la inversión extranjera en el sector agrícola. Esta diversidad de formas económicas responde al objetivo de elevar los niveles de eficiencia y de producción a los fines de potenciar los suministros de alimentos para el pueblo, de materias primas para la industria y de fondos exportables para la generación de MLC20

La reforma económica en curso, iniciada en 1993, constituye un proceso dinámico de profundas transformaciones que abarca a todas las esferas de la economía y la sociedad. Bajo su égida se han modificado la estructura socioeconómica, el mecanismo económico y el funcionamiento del sistema empresarial, con énfasis en la búsqueda de mayores niveles de eficiencia mediante el desarrollo y aplicación de tecnologías blandas y el progreso científico-técnico, en correspondencia con las exigencias del desarrollo sustentable. ${ }^{21}$

En el 2011 se aprueban los Lineamientos de la Política Económica y Social del Partido y la Revolución, como parte de la actualización del modelo económico cubano, con el objetivo el desarrollo económico del país y la elevación del nivel de vida de la población. El modelo reconoce

20 Alemán Santana S.: "La realización socioeconómica de la propiedad en las Cooperativas de Producción Agropecuaria en Cuba (1984-1999)», Tesis de Doctorado, Comisión Nacional de Grados Científicos, Ciudad de La Habana, 2001.

21 VII Congreso del Partido Comunista de Cuba: Lineamientos de la Política Económica y Social del Partido y la Revolución para el período 2016-2021. 
y promueve, además de la empresa estatal socialista, forma principal en la economía nacional, a las modalidades de la inversión extranjera, las cooperativas, los agricultores pequeños, los usufructuarios, los trabajadores por cuenta propia y otras formas que pudieran surgir para contribuir a elevar la eficiencia. ${ }^{22}$

En febrero del 2011 el fondo de tierra ociosa declaradas en el país ascendió a 1868210,84 ha, posteriormente se hace in conteo más intenso y se detectaron 500,0 mil ha, más el fondo de tierras ociosas inicial ascendió a 2,3 millones de ha. Situación que se revirtió en el otorgamiento de esas tierras a aquellos que quisieran trabajarlas, permiso que se tramita a través del Centro Nacional de la Tierra.

\section{La Unidades Básicas de Producción Cooperativa}

Entre los principales problemas a los que en el corto plazo se debe encontrar una solución efectiva y sostenible para el enfrentamiento de los retos planteados al sector agropecuario, está la consecución de una estabilidad de la fuerza de trabajo en las labores primarias demandadas por las diferentes actividades productivas y de apoyo.

Esta meta, la de «sembrar al hombre», como premisa para la reproducción ampliada de la producción agropecuaria en consonancia con las exigencias de la economía nacional, comporta la búsqueda y experimentación de diferentes fórmulas de vinculación del hombre al área, sobre la base de propiciar la permanencia de los trabajadores en el sector, a través del incremento de sus ingresos monetarios y en especies, mejorando su calidad de vida con la construcción de viviendas y facilitando el acceso a otros servicios en el medio rural, a fin de incrementar la productividad y los rendimientos productivos.

Múltiples y variadas fueron las fórmulas experimentadas en décadas, sin que se logrará la estabilización de la fuerza de trabajo y mucho menos la permanencia, sobre todo en el sector de las antiguas empresas estatales. Con la creación de las UBPC se aspiró a superar esta debilidad del modelo de gestión. Es así como entre los cuatro principios básicos se enfatizó la vinculación del hombre al área, el pago por los resultados finales y el mejoramiento de las condiciones de vida de los trabajadores mediante el autoconsumo y las viviendas. ${ }^{23}$

\footnotetext{
22 Ibidem.

23 MINAG: Resumen de los balances económicos de las unidades básicas de producción cooperativa, Cienfuegos, 2015.
} 
En la dirección antes mencionada y en la búsqueda de soluciones, se han desarrollado diversos proyectos con el propósito de introducir y generalizar el modelo de las fincas familiares (también nominadas como integrales) en varias ramas del sector agropecuario, incluyendo la actividad forestal. Este modelo de finca tiene como característica distintiva el otorgamiento de determinadas áreas a los trabajadores y personas naturales para su explotación, correspondiendo a la entidad empresarial (estatal o cooperativa) la comercialización de la parte fundamental de la producción del «finquero», mediante precios acordados previamente en los renglones destinados a la satisfacción del consumo social o del mercado, de destinarse a los mercados de comercialización liberada.

El finquero o responsable de la finca deberá apoyarse básicamente en la fuerza de trabajo familiar para la explotación de la granja, aunque no se excluye la utilización de otras fuerzas no necesariamente con vínculos familiares, sobre todo en los momentos picos de siembra, cosecha, labores culturales emergentes, etc. Esta modalidad de organización productiva de base ha venido reportando resultados muy alentadores desde el punto de vista productivo y socio económico. En la práctica allí donde se ha aplicado esta solución se ha logrado una estabilización de la fuerza de trabajo, configurando gradualmente un paradigma que guarda cierta semejanza con las fincas campesinas predominantes en las cooperativas de crédito y servicios.

En las UBPC estudiadas y en otras a lo largo de la provincia de Cienfuegos donde estas fincas familiares han sido experimentadas como fórmula organizacional, se logran efectos muy positivos en materia de retención laboral, así como una real y efectiva vinculación del hombre al área. Obviamente el camino a recorrer en función del perfeccionamiento de este tipo de mecanismo de incentivación es aún largo, pero lo recorrido hasta la fecha abre expectativas muy favorables y los resultados que se han venido logrando así lo avalan. ${ }^{24}$

En contactos directos con los finqueros durante las visitas realizadas, estos manifestaron su satisfacción y complacencia por la opción elegida. Al preguntársele si la finca la consideraban como suyas, respondieron afirmativamente, pero con la clara conciencia de que para permanecer en ella, era obligatorio cumplir con el reglamente interno de la UBPC, las orientaciones emanadas de la Junta de Administración y los acuerdos de la asamblea general.

24 Ibidem. 


\section{El cooperativismo en la coyuntura actual}

El Sexto Congreso del Partido Comunista de Cuba acuerda aprobar los Lineamientos de la Política Económica y Social del Partido y la Revolución, para actualizar el modelo económico cubano, con el objetivo el desarrollo económico del país y la elevación del nivel de vida de la población. El modelo reconoce y promueve, además de la empresa estatal socialista, forma principal en la economía nacional, a las modalidades de la inversión extranjera, las cooperativas, los agricultores pequeños, los usufructuarios, los trabajadores por cuenta propia y otras formas que pudieran surgir para contribuir a elevar la eficiencia. Se aprueba con el Lineamiento No 168 de la referida Política, ampliar el trabajo en el sector no estatal, como una alternativa más de empleo, en dependencia de las nuevas formas organizativas de la producción y los servicios que se establezcan. En el proceso de actualización del modelo económico cubano es necesario la creación, con carácter experimental, de cooperativas en sectores no agropecuarios, lo que requiere de una norma jurídica que instrumente su creación y funcionamiento ${ }^{25}$.

Como parte de las transformaciones que han venido implementándose en el país desde el VI congreso del Partido, donde se aprueban una serie de lineamientos que responden al orden económico, político y social, se hace énfasis en programas priorizados como el de seguridad alimentaria, cumpliendo con los planes económicos del país proyectados hasta el 2030.

Dentro de los ejes temáticos planteados se encuentran el potencial productivo y el territorial para lograr desarrollar una estrategia coherente e interrelacionada de desarrollo. Lograr una adecuada distribución territorial de las fuerzas productivas, conjugando la dimensión nacional y sectorial con la local, y un desarrollo prospero, ordenado y sostenible, para ello es necesario alcanzar niveles de producción y comercialización agropecuaria que garanticen alto grado de autosuficiencia alimentaria26.

Teniendo en cuenta que en la actualidad cubana uno de los temas que más preocupa al Gobierno y a la población es el sustentos de la canasta básica, sobre todo, en el orden de la alimentación diaria de

25 Resolución sobre los Lineamientos de la Política Económica y Social del Partido y la Revolución. 2018, consultado en https://www.pcc.cu/sites/default/files/congreso/ pdf/20180426/resolucion_sobre_los_lineamientos_de_la_politica_economica_y_social_ del_partido_y_la_revolucion.pdf

26 Documento sobre Principios Rectores y Ejes Temáticos para la elaboración del Plan Nacional de Desarrollo Económico y Social hasta el 2030. 
los cubanos, evitando el lucro, el despilfarro, el sobreprecio de los productos por aquéllos que sirven de intermediarios por cuenta propia, entre el campo y la ciudad; se buscan alternativas para crear una gestión efectiva fundamentalmente en producción, distribución, comercialización y consumo de los alimentos, así como, lograr el encadenamiento productivo, cuyas ventajas benefician a todos los actores implicados.

Desde esta perspectiva la provincia de Cienfuegos también busca nuevas alternativa para la soberanía alimentaria, la sustitución de importaciones, y la efectividad del aparato productivo, y cumpliendo así con el concepto de Revolución proclamado por Fidel Castro Ruz que exhorta a la emancipación con esfuerzos propios. Para ello se debe propiciar una mayor movilidad de las fuerzas productivas hacia el sector agropecuario, para alcanzar mayor nivel de productividad, mediante la diversificación, la modernización tecnológica, la innovación, la participación selectiva de nuevos paradigmas tecnológicos, en particular con un enfoque de alto valor agregado.

Entre los objetivos que se plantea en la visión de la nación para el 2030 está propiciar los encadenamientos productivos hacia el interior del país, elevar la producción, productividad, competitividad y sostenibilidad ambiental y financieras de las cadenas productivas agroalimentarias, a fin de contribuir a la seguridad alimentaria, aprovechar el potencial exportador, incrementar la sustitución eficiente de las importaciones y generar empleos o ingresos particularmente en la población rural, potenciando además con énfasis especial la articulación entre la agricultura y la manufactura, particularmente la industria alimentaria y entre sectores de servicio.

Entre los objetivos de la política económica del estado cubano se encuentra el logro de la seguridad alimentaria nacional, basada en la consolidación de los cambios en la estructura de tenencia y uso de la tierra (descentralizando su uso y disminuyendo el área ociosa con la entrega de tierras estatales en usufructo a distintas formas cooperativas y a particulares, incluidas las zonas montañosas, que han permitido su repoblación), el fomento de la Agricultura Urbana, el desarrollo de fuentes renovables de energía, el uso creciente de la tracción animal, la aplicación de los conceptos de Manejo Integrado de Plagas y de Manejo Integral de la Nutrición (con la consiguiente disminución del uso de agroquímicos), y el manejo de los recursos hídricos sobre la base del Manejo Integrado de Cuencas Hidrográficas.

Entre los antecedentes de estudio de la sostenibilidad de la gestión cooperativa en el sector agropecuario, se encuentra el estudio de la provincia Cienfuegos. Según estos resultados, los aspectos más relevantes de la transformación agraria en el territorio están relacionados 
con la entrega de tierras lo cual provoca el incremento significativo de la cantidad de tenedores en el sector no estatal, la reorganización del sector empresarial y uso de suelos, la disminución del uso de insumos externos respecto al período anterior a la crisis, el uso de alternativas tecnológicas para la producción vegetal y animal, el fomento de la agricultura urbana y periurbana, el incremento de la diversidad de canales de comercialización, el ajuste de estructuras institucionales y un grupo de limitantes que tienen como expresión final la insuficiencia de la producción local de alimentos. ${ }^{27}$

Según los resultados de este trabajo, los factores limitantes fundamentales de la sostenibilidad de la transformación agraria en la provincia se definen como la ineficiencia de la gestión agraria junto al bajo per cápita de la producción mercantil agropecuaria, la relación no equilibrada de la productividad / ingreso medio, la alta relación población urbana: población rural y la baja proporción de la población económicamente activa agropecuaria, consecuencias básicamente de los problemas en la disponibilidad, uso y distribución del agua; bajo porcentaje de la superficie total bajo riego y riego de alta tecnología y el déficit de energía e insumos para la producción de alimentos. ${ }^{28}$

Las cooperativas de producción agropecuarias históricamente han contribuido al desarrollo local de los territorios en los que se encuentran enclavadas, asistiendo de manera general al bienestar de sus poblaciones. Sin embargo, el movimiento cooperativo en el actual proceso de internacionalización, está pasando por una transformación importante cuya misión, ideología y desempeño en el medio rural, está alejándose de los principios históricos. Los objetivos y estrategias seguidas por numerosas cooperativas respaldan la introducción de métodos de gestión identificados con la dogmática lógica empresarial y de mercadeo, y que tiende a privilegiar al gran productor comprometido con una agricultura intensiva y productora de mercancías destinadas, principalmente, al abastecimiento del mercado externo.

Desde esta visión, las cooperativas a pesar de seguir fines meramente sociales debido al vínculo con el estado en el caso de Cuba, debe de tener un mayor sentido de partencia, y crear programas más actualizados para lograr incorporar el universo juvenil a sus filas, sin

27 Socorro, A.: Indicadores de la sostenibilidad de la gestión agraria en el territorio de la provincia de Cienfuegos. 2002. Universidad Agraria de la Habana.

28 Acosta Morales, Y. y Sánchez Quintero, M.: «Seguridad alimentaria en Cuba en la coyuntura actual: fincas familiares y cooperativas sostenibles». Revista Científica Agroecosistemas, 7(3). 2019. pp. 142-147. 
perder de vista sus principios fundamentales y en aras de la formación de valores.

Relacionado a esto, la Alianza Cooperativa Internacional expresa que: "Toda cooperativa que implícita o explícitamente renuncia a resolver los problemas del conjunto de su base social o las relaciones con los socios-agricultores, se limita únicamente a un mero trato comercial y terminan a la larga transformándose en otras sociedades diferentes a las cooperativas».

En el mundo actual las cooperativas agrarias pasan a funcionar como empresas globales, a gran escala, subordinadas fundamentalmente a los intereses del mercado, controladas por gestores que paulatinamente se divorcian de la base social, y en donde aspectos como el bienestar de todos los socios, la solidaridad, o la atención a la comunidad, son sacrificados en beneficio de otros intereses como la buena gestión y la eficacia económica, que estrictamente no tienen por qué coincidir con los cooperativos. Desde esta visión más empresarial se erigen cooperativas cuya estrategia y orientación adquieren un discurso más reivindicativo y comprometido socialmente, y apuestan por una cooperativa agraria que sirva como instrumento colectivo de defensa y reivindicación de los intereses de las clases sociales del campo menos favorecidas. En este caso el desarrollo de la producción debe servir para aumentar el bienestar social de los socios, las familias y comunidad, y no estar al servicio del incremento patrimonial de una parte de su base social.

La sostenibilidad es imprescindible para referirnos a la capacidad de satisfacer las necesidades actuales de la población, sin poner en riesgo las de generaciones futuras. Por lo tanto, en materia de cooperativismo agroalimentario, se trataría de establecer actividades dentro de un programa de sostenibilidad de la industria de base cooperativa, en sus tres formas, sostenibilidad ambiental, económica y social29.

\section{Conclusiones}

Las cooperativas agropecuarias tienen una superioridad competitiva de partida, como es la sostenibilidad social. El mantenimiento de la cohesión social tanto dentro como fuera de la empresa, fortaleciendo las relaciones y estableciendo compromisos perdurables con los socios, los

29 Puentes, R. y Velasco, M.: «Importancia de las Sociedades Cooperativas como medio para contribuir al desarrollo económico, social y medioambiental, de forma sostenible y responsable» (pp. 104-129). REVESCO n. ${ }^{\circ} 99$. Tercer cuatrimestre 2009, consultado en www.ucm.es/info/revesco 
ciudadanos y la comunidad rural es una marca diferencial de las cooperativas agroalimentarias. La estabilidad en el empleo, la promoción interna y la conciliación laboral y personal, así como de las actividades vinculadas al aspecto cultural y tradicional de una zona (gastronomía, producciones y factores de producción protegidos, sostenibilidad alimentaria en el consumo responsable) son hechos que se suelen vincular a las cooperativas.

Estas entidades estarán contribuyendo al desarrollo local desde los territorios, en sus aspectos sociales, económicos y ambientales según la estrategia de desarrollo hasta el 2030, en relación con un crecimiento integrador, sostenible e inteligente, que disfrute de altos niveles de empleo, de productividad y de cohesión económica, social y territorial. Por otra parte, en la búsqueda de un modelo económico más sostenible, el cooperativismo se erige como una alternativa competitiva y eficiente, que ofrece nuevas respuestas a los desequilibrios de la cadena de valor del sector agroalimentario y que, a su vez, promueve el empleo y promueve las cadenas alimentarias locales, la seguridad alimentaria, la participación y la responsabilidad de toda la sociedad.

\section{Bibliografía}

1. Acosta Morales, Y., \& Sánchez Quintero, M.: «Seguridad alimentaria en Cuba en la coyuntura actual: fincas familiares y cooperativas sostenibles». Revista Científica Agroecosistemas, 7(3). 2019. pp. 142-147.

2. Alemán Santana, S.: La realización socioeconómica de la propiedad en las Cooperativas de Producción Agropecuaria en Cuba (1984-1999), Tesis de Doctorado, Comisión Nacional de Grados Científicos, Ciudad de La Habana, 2001.

3. Altuna Gabilondo, L.; Loyola Idiakez, A.; Pagalday Tricio, E.: Mondragón: los dilemas de un cooperativismo maduro. Editorial Caminos, Cuba. 2012.

4. DÁVILA, G. Innovación y éxito en la gerencia cooperativa: Casos exitosos de cooperativas rurales de ahorro y crédito. Bogotá: Pontificia Universidad Javeriana. 2002.

5. GOYES, S., y LÓPEZ, V.: Experiencias empresariales exitosas: factores de éxito empresarial en las fases de emprendimiento, gestión y supervivencia. San Juan de Pasto: Institución Universitaria Centro de Estudios Superiores María Goretti. 2006.

6. UNIRCOOP: Éxito e innovación en la gestión: las cooperativas como agentes del desarrollo local. 2005.

7. Alfonso SÁnchez, R.: La integración cooperativa y sus técnicas de realización: La cooperativa de segundo grado. Valencia: Editorial Tirant lo blanch. 574 p. ISBN: 84-8442-120-1. 2000. 
8. Bertullo et al.: "El cooperativismo en Uruguay». Documento de Trabajo del Rectorado N. ${ }^{\circ}$ 22, Universidad de la República. Montevideo. 2004.

9. Burdín, G. y Dean, A.: "Las cooperativas de trabajo asociado en Uruguay. Caracterización y evolución en el período 1996-2005. Avance de investigación». Centro de Investigaciones para el Desarrollo Internacional de Canadá (IDRC). Cooperativas e Integración Regional MERCOSUR. Uruguay. 2006.

10. Castro Ruz, F.: Discurso Clausura V Encuentro Internacional de Economistas sobre Globalización y Problemas del Desarrollo. La Habana. 2003.

11. Castro Ruz, R.: Discurso pronunciado en el Aniversario del Asalto al Cuartel Moncada. Periódico Granma (28-7-07). La Habana. 2007.

12. ChÁvez FríAS, H. Discurso en el II Foro Social Mundial. Porto Alegre. Brasil. 2003.

13. Cruz Reyes, J. y Piñeiro Harnecker, C.: La introducción a las cooperativas. Editorial Caminos. La Habana. 2012.

14. CRUz REYES, J.: «Sobre cooperativas agrícolas de segundo grado: experiencias cubanas y consideraciones hacia el futuro». Economía y DesarroIlo, vol. 158, núm. 1, enero-junio, Universidad de La Habana La Habana, Cuba. 2017. pp. 225-244.

15. Cuenca García, Á.: «Las cooperativas de segundo grado en la Ley 27/1999 de 16 de julio de cooperativas». Revista Jurídica de Economía Social y Cooperativa, CIRIEC-España, n. ${ }^{\circ}$ 11. 2000.

16. DÁvILA, M. et al.: «Éxito e innovación en la gestión: las cooperativas como agentes del desarrollo local». Red uniRcoop. Nodo Andino. Pontifica Universidad Javeriana. Colombia. 2005.

17. DAVIS, P.: «La administración de la diferencia cooperativa». División Cooperativa-OIT. Suiza. 1999.

18. Documento sobre Principios Rectores y Ejes Temáticos para la elaboración del Plan nacional de Desarrollo Económico y Social hasta el 2030.

19. Federación de Cooperativas de Producción del Uruguay, «Manual básico para cooperativas de trabajo asociado de la FCPU». 2010.

20. Fernández Peiso, A.: La Contabilidad en el Sector Cooperativo. Protección Legal, Informe. 2001.

21. Fernández Peiso, A.: La Cooperativa. Bases para su legislación en Cuba. La Habana: Ciencias Sociales. 2012.

22. Figueroa Albelo, V.: UBPC, Desarrollo rural y participación, El nuevo Modelo Agrario en Cuba bajo los Marcos de la Reforma Económica, Ed. U. H. La Habana. 1998.

23. Franguelul, F. Reflesi della: IV Directiva CEE sui bilanci delle società cooperative, Ed. TECNOSTUDI, Roma. 1995.

24. Fray Beto: Ley en Porto Alegre». Periódico Juventud Rebelde. 25 de enero. La Habana. 2002.

25. García Muller, A.: "Marco Jurídico de la Economía Solidaria en Latinoamérica». Anuario de Estudios Cooperativos 1998. Ed. A. Mugarra. Bilbao. 1999. 
26. Hudson, J. P.: «Políticas públicas y empresas recuperadas por sus obreros en Argentina: un análisis del Programa de Trabajo Autogestionado 20042012». Apuntes, 43 (79). 2016. pp. 157-184.

27. MARTí, J. P. et al.: «Cooperativas de trabajo en el Cono Sur. Matrices de surgimiento y modelos de gestión». II Jornada Universitaria sobre Cooperativismo, Economía Solidaria y Procesos Asociativos. Documento de Trabajo del Rectorado, No. 30. UdelaR. Uruguay. 2006.

28. MarX, C.: Obras escogidas. TII. Ed. Progreso. Moscú. 1989.

29. Miranda LoRenzo, Humberto: TEMA 3. Cooperativismo y autogestión en la visión de Marx, Engels y Lenin. Parte 2. Las cooperativas y los pensadores socialistas. Cooperativas y socialismo. Una mirada desde Cuba. 2012.

30. Moreno, D.: «El nuevo régimen jurídico de las cooperativas en Uruguay». Revista Estudios Cooperativos. Año 14-N. ${ }^{\circ}$ 1. Setiembre. Unidad de Estudios Cooperativos-UdelaR. Montevideo. 2009, pp. 76-89.

31. Morrillas Jaramillo, M. J. y Feliú Rey, M.I.: Curso de Cooperativas. Madrid. Editorial Tecnos, s/f.

32. NovA, A.: Las cooperativas agropecuarias en Cuba: 1959-presente. Editorial Camino. 2012.

33. Pineda Suárez, C.J.; Pardo Martínez, e., Castillo Soto, M,E.; Palacios VilleGAS, N.: Cooperativismo mundial 150 años. Consult América Grupo Asesor. Bogotá, Colombia. 1994.

34. MINAG: Resumen de los balances económicos de las unidades básicas de producción cooperativa, Cienfuegos. 2015.

35. Rojo Ramírez, A.: La fusión de sociedades anónimas en la reforma de la Ley de Sociedades Anónimas. Madrid: Editorial Cívitas. 1987.

36. SOCoRro, A.: Indicadores de la sostenibilidad de la gestión agraria en el territorio de la provincia de Cienfuegos. Universidad Agraria de la Habana. 2002.

37. ValdÉs PAZ, J.: Los procesos de organización agraria en Cuba 1959-2006. Fundación Antonio Núñez Jiménez de la Naturaleza y el hombre, La Habana, 2010.

38. Vieitez Giraldez, C. y Dal Ri, N.: «Trabalho Associado. Cooperativas e empresas de autogestao». DP\&A editora. Bras. 2001. 


\section{Derechos de autor}

La revista Deusto Estudios Cooperativos es una revista de acceso abierto lo que significa que es de libre acceso en su integridad inmediatamente después de la publicación de cada número. Se permite su lectura, la búsqueda, descarga, distribución y reutilización legal en cualquier tipo de soporte sólo para fines no comerciales y según lo previsto por la ley; sin la previa autorización de la Editorial (Universidad de Deusto) o el autor, siempre que la obra original sea debidamente citada (número, año, páginas y DOI si procede) y cualquier cambio en el original esté claramente indicado.

\section{Copyright}

The Deusto Journal of Cooperative Studies is an Open Access journal which means that it is free for full and immediate access, reading, search, download, distribution, and lawful reuse in any medium only for non-commercial purposes, without prior permission from the Publisher or the author; provided the original work is properly cited and any changes to the original are clearly indicated. 